\title{
A GENERAL FORMALISM FOR QUASI-LOCAL CORRECTION OF MULTIPOLE DISTORTIONS IN PERIODIC TRANSPORT SYSTEMI*
}

\author{
David Neuffer \\ Los Alamos National Laboratory \\ AT-6 \\ Los Alamos, New Mexico 87545
}

\author{
Etienne Forest \\ Accelerator \& Fusion Research Division \\ Lawrence Berkeley Laboratory \\ University of California \\ Berkeley, California 94720
}

June 1988

\begin{abstract}
DISCLAIMER
This report was prepared as an account of work sponsored by an agency of the United States Government. Neither the United States Government nor any agency thereof, nor any of their employees, makes any warranty, express or implied, or assumes any legal liability or responsibility for the accuracy, completeness, or usefulness of any information, apparatus, product, or process disclosod, or represents that jts use would nol infringe privately owned rights. Refer. ence herein to any specific commercial product, process, or service by trade name. trademark, manufacturer, or olherwise does not necessarily constitute or imply its endorsement, recommendation, or favoring by the Unitud States Government or any agency thereof. The views and opinions of authors expressed herein do not necessarily state or reflect those of the United Stales Government or any agency thereof.
\end{abstract}

*This work was supported by the Office of Energy Research, Office of Basic Energy Sciences, Department of Energy under Contract No. DE-AC03-76SF00098. 
A General Formalism for

Quasi-Local Correction of Multipole Distortions in Periodic Transport Systems

\author{
DAVID NeUfFer \\ AT-6 Los Alamos National Laboratory \\ Los Alamos, NM 87545 \\ Etienne Forest \\ Exploratory Studies Group \\ Accelerator Fusion Research Division \\ Lawrence Berkeley Laboratory \\ Berkeley, CA 94720
}

June 1988

\begin{abstract}
We generalize a new concept of local correction of nonlinearities due to multipole content by giving it a mathematical description. We present a general method which allows for a general reduction of all the distortions produced by a given set of multipole errors. The method can be applied to correct an arbitrary distribution of we errors in any travisport system, such as transport lines, linacs,synchrotrons and storage rings.
\end{abstract}


For reasons is stability and reliability, predominantly linear motion is required in a long beam transport system. In this linear motion, particle trajectories lie on ellipses in position-momentum phase space. Nonlinear motion due to multipole fields changes these trajectories in both amplitude and phase, distorting the phase space and causing instability on short or long time scales $[1,2]$. Therefore, one must correct the nonlinear effects of the multipole content present in the various elements of the system. A common correction technique for short optical systems (mass spectrometers or electron microscopes) is to identify a few key distortions and insert lumped correctors specifically designed to eliminate those. However, in long periodic transport, systems, nonlinear effects can accumulate either coherently or noncoherently over repeated cells and a large number of distortions can affect the beam. Hence the nonlinear effects cannot be cancelled by a lumped correction scheme without either a prohibitively large number of correctors or undesirable high order effects due to large corrector strengths. In this paper, we present a new method of locally correcting nonlinearities in each transport cell in a way which reduces all nonlinear effects due to a given multipole content.

Figure 1 shows schematically a cell of a periodic transport system. Regions I and 3 are linear focussing or defocussing elements. Region 2 between those elements contains an arbitrary transport element with significant multipole content. The linear motion is represented by a Hamiltonian $H(s)$ which has two parts: a sindependent part $H_{0}$ which controls the motion in region 2 and an additional sdependent part $U(s)$ which differs from $H_{0}$ in the focussing regions 1 and 3 .

$$
H(s)=H_{0}+U(s)
$$

Let us assume that the Hamiltonian is modified by a multipole represented by a term $e(s) W$, where $e(s)$ is the known multipole distribution throughout region 2, 
and $W$ is the Hamiltonias perturbation due to that multipole. We counteract its effect with a corrector distribution $c(s)$ of the same multipole. The trivial correction solution is given by

$$
c(s)=-e(s)
$$

We refer to this solution as "local" correction; it is not practical in general. Instead, we derive conditions on $\mathrm{c}(\mathrm{s})$ which achieves a close approximation to Eq.(2).

In region 2 , the motion is governed by the Hamiltonian $K(s)$ :

$$
K(s)=H_{0}+(e(s)+c(s)) W
$$

Following Dragt's notation for the Lie operators [3]parametrizing a beam transport, we can write a map $\mathcal{M}$ which transforms any function of phase space from the beginning of region 2 at $s=0$ to its end at $s=L$. To second order order in $W, \mathcal{M}$ is given by [4]:

$$
\begin{gathered}
\mathcal{M}=\exp \left(: w_{2}:\right) \exp \left(: w_{1}:\right) \exp \left(:-L H_{0}:\right) \ldots \\
w_{1}=\int_{0}^{L} \exp \left(:-s H_{0}:\right)(e(s)+c(s)) W d s \\
u_{2}=\frac{1}{2} \int_{0}^{L} \int_{0}^{b}\left[\exp \left(:-a H_{0}:\right)(e(a)+c(a)) W, \exp \left(:-b H_{0}:\right)(e(b)+c(b)) W\right] d b d a .
\end{gathered}
$$

We expand the function $w_{1}$ in powers of the Lie operator $H_{0}$ :

$$
\begin{aligned}
& w_{1}=\sum_{n=0}^{\infty} F_{n} \frac{:-H_{0}:^{n} W}{n !}, \\
& F_{n}=\int_{0}^{L}(e(s)+c(s)) s^{n} d s .
\end{aligned}
$$

The $F_{n}$ are the $n^{\text {th }}$ moments of tise total multipole distribution in region 2 . We call a 
system that sets all $F_{n}$ to zero for $n \leq m-1$ a m-parameter quasi-local correction system. We re-emphasize the underlying assumptions behind this approach and contrast it with "lumped" correction. The region 2 in which errors are cancelled is a constant unperturbed Hamiltonian $\left(H_{0}\right)$ region; this is essential if the expansion of equation (5a) is to be valid. We have also implicitly assumed that the form factors : $-H_{0}:^{n} W$ are well-behaved as $n$ increases; this is generally valid where $H_{0}$ is either a drift or a bend.

An important advantage of quasi-local correction is that its ability to reduce the multipole distortions is not affected by deviations in the focussing potential $U(s)$. By contrast. lumped correction uses correctors which are far from the corrected multipole cc:itent (i.e. separated by one focussing regions) to eliminate a few distortions. Their strengths are dependent on $U(s)$; changes in in $U(s)$ can greatly deteriorate the correction. This sensitivity is greatly increased as the length of the beam transport and the number of nontrivial nonlinear effects increases.

In practice $c(s)$ will consist of short elements and in the examples presented below they are represented by zero length correctors of integrated strength $C_{i}$ at locations $s_{i}$ :

$$
c(s)=\sum_{i=1}^{m} C_{i} \delta\left(s-s_{i}\right) .
$$

All the exaunples use a simple FODO cell with six sector bends per half cell. We display results for three types of errors in the bends: normal sextupole, normal octupole and normal decapole $\left(b_{2}, b_{3}\right.$, and $b_{4}$ respectively). The results are indicative of the type of correction achievable by quasi-local correction.

In the first example, we consider a constant $e(s)=E_{0}$ in region 2 . For $\mathrm{m}$ correctors equally spaced from $s=0$ to $s=L$, the weights $C_{j}$ are given by the Newton-Cotes $[5](\mathrm{NC})$ integration strengths. For $m=3$ we obtain the Simpson's 
Rule values $\left(C_{1}=C_{3}=\frac{-L E_{0}}{6} ; C_{2}=\frac{-4 L E_{0}}{6}\right)$, as shown in Figure 2. To compare the various configurations, we use a norm for the Lie polynomials $w_{1}$ and $u_{2}$ of the full cell [6]. The uncorrected configuration has norm one. In table 1, a dramatic improvement is obtained in going from $m=2$ to $m=3$. In general, one obtains one extra order of accuracy from symmetry when $m$ is odd and $c(s)$ is constant (or symmetric about $s=L / 2)$ in a $\mathrm{NC}$ geometry. Also, to show the dependence on proper selection of the corrector strengths, we display the results of a three-point equalweight scheme. Inprovement is also obtained in the second order nonlinearities $w_{2}$, which occurs because the methods are quasi-local.

The case $m=2$ corresponds to placing the correctors near the focussing elements, which has previously been the usual practice. The case $m=3$ adds a corrector at the center of the transport cell and provide great improvements over previous methods $[7,8,9]$.

If the focussing potential $U(s)$ nearly commutes with the potential $W$, it is possible to use $m-1$ actual correctors and obtain an m-parameter NC correction by combining the last corrector of one constant $H_{0}$ region with the firsi one of the following region. This can be extended to any distribution of errors and correctors provided that a corrector is very near the focussing region. In practice, $W$ nearly commutes if $U^{\prime}(s)$ represents a sufficiently thin quadrupole.

In the sccond example [table 2], we consider again a constant $c(s)$ but optimize the locations as well as the strengths of the correctors. In this case the weights $C_{i}$ and the positions $s_{i}$ are given by Gaussian Quadrature (GQ) values [5]. Choosing the positions $s_{i}$ to zero extra moments is not always feasible; lowever the possibility of optimizing these positions should be considered. For the same $m$, the GQ method is better in our example than the corresponding NC configuration. This is not a general result; it depends on the nature of the cell and its errors. 
In the previous examples, the case $e(s)=E_{0}$ was used. The method is easily extended to include $\mathrm{e}(\mathrm{s})$ varying throughout region 2 with similar improvements in linearity. For example [see figure 3], we consider a region with six independent errors which are corrected by an equally spaced arrangement. The optimal strengths for the correction are:

$$
\begin{aligned}
& C_{1}=\frac{L}{648}\left(-83 e_{1}-41 e_{2}-11 e_{3}+7 e_{4}+13 e_{5}+7 e_{6}\right), \\
& C_{2}=-\frac{L}{81}\left(4 e_{1}+10 e_{2}+13 e_{3}+13 e_{4}+10 e_{5}+4 e_{6}\right), \\
& C_{3}=\frac{L}{648}\left(7 e_{1}+13 e_{2}+7 e_{3}-11 e_{4}-41 e_{5}-83 e_{6}\right) .
\end{aligned}
$$

If all $e_{i}$ are equal, we regain the NC formula. Statistical computation and tracking results confirm the validity of this correction [10].

If $e(s)$ varies from cell to cell, the strengths $C_{i}$ should also vary from cell-to-cell. However if a GQ-like algorithm is employed, the corrector locations must also vary from cell-to-cell. That may be impractical in a repeating cell structure.

We have outlined an extremely powerful method of quasi-local correction of multipole content in a long transport system. The method uses discrete correctors powered so as to locally cancel the lowest-order moments of that multipole content. In the simplest case of constant multipole fields, the solutions can be expressed in tcrms of finite-point integration formulae. The full method can provide enormous reduction in all nonlinear effects, and in all orders.

We acknowledge stimulating discussions with J. M. Peterson, P. Channell, R. Talman and A. Chao. 


\section{References}

1. R. Ruth, in Physics of Particle Accelerators, Ed. by M. Month and M. Dienes.

2. M. Michelotti, in Ref. 1, p.260 .

3. A.J. Dragt and E. Forest, J. Math. Phys. 24,2734 (1983).

4. The derivation of this result uses a differential equation for the symplectic map $\mathcal{M}$ and the concept of the Dirac representation (ref. 3). See A. Messiah, Mecanique Quantique, p.270 (Dunod, 1965) for a familiar quantum mechanical version.

5. Handbook of Mathematical Functions, National Bureau of Standards, Washington, DC, (1972).

6. The norm $\sigma\left(w_{i}\right)$ of $w_{i}$, the $i^{\text {th }}$ order Lie polynomial in $e(s)$, is computed by transforming $w_{i}$ into normalized variables and adding the radii of the resonance driving terms:

$$
\begin{gathered}
w_{i}=\sum_{a b c d}\left(A_{a b c d}^{i} \cos \left(c \phi_{x}+d \phi_{y}\right)+B_{a b c d}^{i} \sin \left(c \phi_{x}+d \phi_{y}\right)\right) J_{x}^{\frac{a}{2}} J_{y}^{\frac{b}{2}} \\
\sigma\left(w_{i}\right)=\sum_{a b c d}\left(A_{a b c d}^{2}+B_{a b c d}^{2}\right)^{\frac{1}{2}}
\end{gathered}
$$

This norm is a good figure of merit whenever the system is periodic and equal $J_{x}$ and $J_{y}$ are expected.

7. D. Neuffer, Particle Accelerators, Vol. 23, p. 21, (1988)

8. D. Neuffer, Los Alamos Tech. note LA-UR-87-3903, submitted to Nuclear Instruments and Methods. 
9. In these initial applications (Refs. 4 and 5), correction by more than two orders of magnitude are obtained in the critical non-linearities of trajectory dependent phase shifts, which add coherently from cell to cell.

10. E. Forest and J. M. Peterson,"Correction of Random Multipue Errors with Lumped Correctors", to be published in the European Accelerator Conference proceedings, Rome, (1988).

11. MARYLIE 5.0, a fifth order analysis code of the MARYLIE family, was used in the computation of the $\sigma\left(w_{i}\right)$ 's. F. Neri and A. J. Dragt at the University of Maryland, (unpublished).

\section{Figure Captions}

Figure 1: A transport cell with short focussing elements ( $I$ and 3 ) and ong transpurt regions (2).

Figure 2: Three-point Newton-Cotes-like correction of a constant error $E_{0}$, and two-point Gaussian-Quadrature-like correction.

Figure 3: A transport segment with six independent error elements and three correctors. 
Table 1: Newton-Cotes-like correction of sextupole, octupole and decapole.

\begin{tabular}{|c|c|c|c|c|c|c|c|}
\hline \multirow[b]{2}{*}{$\begin{array}{l}\text { corrector } \\
\text { strengths }\end{array}$} & \multirow[b]{2}{*}{$\mathrm{m}$} & \multicolumn{3}{|c|}{$\sigma\left(w_{t}\right)$} & \multicolumn{3}{|c|}{$\sigma\left(w_{2}\right)$} \\
\hline & & $b_{2}$ & $b_{3}$ & $b_{4}$ & $b_{2}$ & $b_{3}$ & $b_{4}$ \\
\hline$(1 / 2,1 / 2)$ & 2 & .54 & .68 & .83 & .62 & 1.0 & $\cdots$ \\
\hline$(1 / 6,4 / 6,1 / 6)$ & 3 & .17 & .14 & .20 & .074 & .09 & $\cdots$ \\
\hline$(1 / 8,3 / 8,3 / 8,1 / 8)$ & 4 & .13 & .11 & .15 & .039 & .045 & $\cdots$ \\
\hline & & & Ial $w$ & his 3 & orrecto & scheme & \\
\hline$(1 / 3,1 / 3,1 / 3)$ & 2 & .35 & .40 & .50 & .26 & .38 & $\cdots$ \\
\hline
\end{tabular}


Table 2: Gaussian Quadrature-like correstion of sextupole, octupole and decapole.

\begin{tabular}{|c|c|c|c|c|c|c|c|}
\hline \multirow{2}{*}{$\begin{array}{l}\text { corrector } \\
\text { strenghts }\end{array}$} & & \multicolumn{3}{|c|}{$\sigma\left(w_{1}\right)$} & \multicolumn{3}{|c|}{$\sigma\left(w_{2}\right)$} \\
\hline & $m$ & $b_{2}$ & $b_{3}$ & $b_{4}$ & $b_{2}$ & $b_{3}$ & $b_{4}$ \\
\hline$(1 / 2,1 / 2)$ & 4 & .0016 & .0088 & .01 & .036 & .038 & $\cdots$ \\
\hline$(5 / 18,8 / 18,5 / 18)$ & 6 & .0098 & .008 & .0097 & .018 & .019 & $\cdots \cdot$ \\
\hline
\end{tabular}




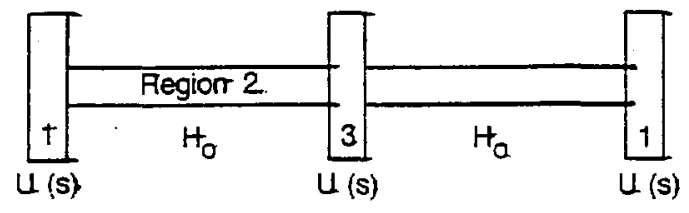

Figure 1
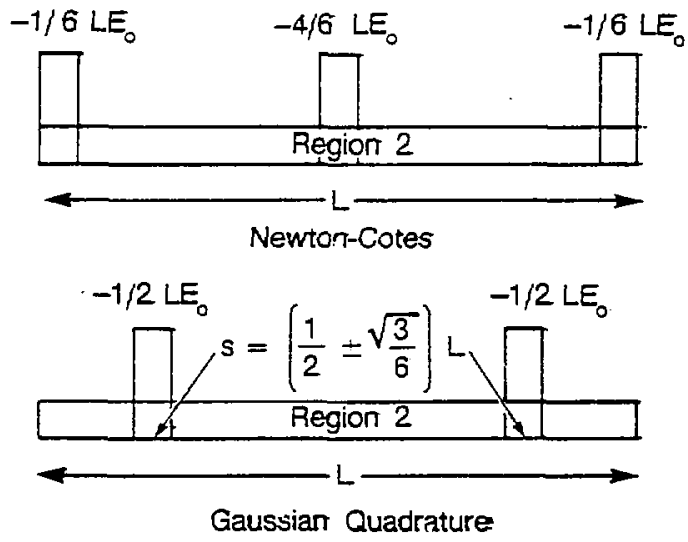

Figure 2

XBL 886-9662 


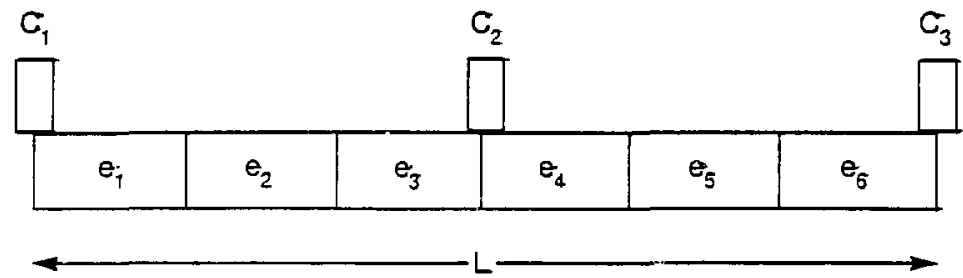

Figure 3

XBL 886-9679 